\title{
View-Dependent JPEG 2000-based Mesh Streaming
}

\author{
Nien-Shien Lin*
}

\author{
Ting-Hao Huang* \\ National Taiwan University
}

\author{
Bing-Yu Chen ${ }^{\dagger}$
}

\section{Introduction}

For PC and even mobile devices, video and image streaming technologies, such as H.264 and JPEG/JPEG 2000, are already mature. However, the 3D model streaming technology is still far from practical use. Therefore, we wonder if 3D model streaming can directly benefit from current image and video streaming technologies. Hence, in this poster, we propose a mesh streaming method based on geometry image [Gu et al. 2002] and integrate it into an existed client-server multimedia streaming server. In this method, the mesh data of a 3D scene is first converted into a JPEG 2000 $(\mathrm{J} 2 \mathrm{~K})^{1}$ image. Based on the JPEG 2000 streaming technique, the mesh data can then be transmitted as a mesh streaming. Furthermore, the view-dependent issue is also taken into account. Since we encode the 3D scene in a JPEG 2000 image, it is much suitable to be integrated into any existed image and video streaming system.

\section{JPEG 2000-based Mesh Streaming}

To convert a 3D model into a JPEG 2000-based mesh streaming, we first convert the model into its geometry image [Gu et al. 2002]. Then, we encode the geometry image as a JPEG 2000. Finally, the $3 \mathrm{D}$ model can be transmitted as a mesh streaming by using the progressive compression option of JPEG 2000. By using the region of interest (ROI) option of JPEG 2000, the portion of a model which is more important will be coded more precisely or earlier in the bit stream. Furthermore, the JPEG 2000 code stream can also be rearranged depending on the user's viewpoint for view-dependent approach. In theory, any compression method could be applied to the scene image to reduce the image size, however, the reasons we adopt JPEG 2000 format are:

- JPEG 2000 supports block-based compression, and our viewdependent method is also block-based algorithm.

- JPEG 2000 has a higher compression ratio even used for lossless compression compared with traditional JPEG format. According to our experiment result, lossy image compression method, such as JPEG, cannot be applied in our system since the reconstructed 3D model will be distorted tremendously, even with the highest quality setting.

- JPEG 2000 supports multi-layer compression, which we could compress the geometry image, normal maps, texture image, texture coordinates, etc. into a single JPEG $2000 \mathrm{im}-$ age.

Besides transmitting the JPEG 2000-based mesh streaming sequentially, we also take the view-dependent issue into account. By using view-dependent mesh streaming, the user can first get a coarse scene in a short time from his/her current viewpoint and then refine the details of the scene.If the user change the viewpoint of the scene, the details of the previous viewpoint are not refined any more, but the region of the current viewpoint will be transmitted.

\footnotetext{
*e-mail:\{ppbb,richardg\}@cmlab.csie.ntu.edu.tw

†e-mail:robin@ntu.edu.tw

${ }^{1}$ http://www.jpeg.org/jpeg2000/
}

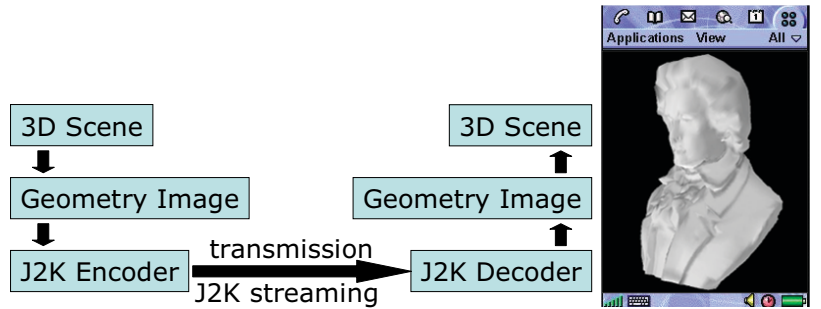

Figure 1: Left: System architecture; Right: Rendering result on a mobile phone.

For transmitting the JPEG 2000-based mesh streaming with the view-depend support, the server first divides the JPEG $2000 \mathrm{im}$ age (geometry image) into several blocks, which is our basic unit in transmission. The block size is directly proportional to the resolution of the image. While obtaining the block stream, the client transmits the viewing information of the current frame to the server when the user changed the viewpoint. Then, the server re-arranges the sequence of candidate blocks of the scene based on the viewing information and the blocks near the candidate blocks are arranged as a spiral-manner sequence. The system architecture is shown in Figure 1 Left.

\section{Decoder on Mobile Devices}

Our JPEG 2000-based mesh streaming approach is suitable for mobile devices. First, since the screen size of mobile devices is relatively smaller than PCs, a smaller geometry image is enough for representing the original 3D model and a distorted model due to higher image compressing rate is also acceptable on the mobile devices. Second, the DSP chip on the mobile devices can be used for the decoder of the mesh streaming, since it is designed to decode image and video streaming. In our implementation, we use J2ME and $\mathrm{M} 3 \mathrm{G}$ as our development environment, so that our program can be run on most mobile devices. The result on a mobile device is shown in Figure 1 Right.

\section{Result and Conclusion}

In most cases, we use a $256 \times 256$ JPEG 2000 image to represent a 3D model. The file size of the image is only 33\% 40\% of the original 3D model with an acceptable visual result. In conclusion, we proposed a new mesh streaming method based on a mature image streaming method directly. Using our method, any client-server system that supports JPEG 2000 can be adapted to transmit 3D models with minimum modifications.

\section{References}

Gu, X., Gortler, S. J., ANd Hoppe, H. 2002. Geometry images. ACM Transactions on Graphics (SIGGRAPH 2002 Conference Proceedings) 21, 3, 355-361. 